\title{
Exoskeleton-based robotic platform applied in biomechanical modelling of the human upper limb
}

\author{
Andres F. Ruiz*, Eduardo Rocon and Arturo Forner-Cordero \\ Bioengineering Group, Instituto de Automatica Industrial 28500 Arganda del Rey, Madrid, Spain
}

(Received 7 October 2008; final version received 15 December 2008)

\begin{abstract}
One of the approaches to study the human motor system, and specifically the motor strategies implied during postural tasks of the upper limbs, is to manipulate the mechanical conditions of each joint of the upper limbs independently. At the same time, it is essential to pick up biomechanical signals and bio-potentials generated while the human motor system adapts to the new condition. The aim of this paper is two-fold: first, to describe the design, development and validation of an experimental platform designed to modify or perturb the mechanics of human movement, and simultaneously acquire, process, display and quantify bioelectric and biomechanical signals; second, to characterise the dynamics of the elbow joint during postural control. A main goal of the study was to determine the feasibility of estimating human elbow joint dynamics using EMG-data during maintained posture. In particular, the experimental robotic platform provides data to correlate electromyographic (EMG) activity, kinetics and kinematics information from the upper limb motion. The platform aims consists of an upper limb powered exoskeleton, an EMG acquisition module, a control unit and a software system. Important concerns of the platform such as dependability and safety were addressed in the development. The platform was evaluated with 4 subjects to identify, using system identification methods, the human joint dynamics, i.e. visco-elasticity. Results obtained in simulations and experimental phase are introduced.
\end{abstract}

Keywords: human motor control; exoskeletons; modelling; biomechanics; elbow joint dynamics

\section{Introduction}

Understanding and modelling the human motor system has become very important and requires the ability to simultaneously record sensory and motor responses during different motor tasks. Biomechanical modelling and analysis of human motion are central topics of interest for a number of disciplines, ranging from biomechanics to human movement science. In addition, during recent years there has been a growing interest in the design and development of robotic devices and artificial tools, in the form of prosthetic and orthotic systems, with functional dynamics that resemble human ones in order to allow the control to be as natural as possible.

Thus, sensing and actuation systems as well as control strategies have been inspired biologically with models that capture the main features of the human motor control system (Kazerooni 1990; Herr et al. 2003). This understanding about the human motor system permits to develop innovative bio-inspired control strategies to be implemented in devices that interface with the human body, for instance prostheses and orthoses, as well as to explore new therapies in disabled people with pathologies and disorders affecting the motor system.

In literature, numerous studies have modelled the dynamic behaviour of human body segments as a mechanical impedance, (Hogan 1985; Dolan et al. 1993; Tsuji et al. 1995). Mechanical impedance in this context may be defined as the dynamic relationship between forces and position variations. In fact, the modulation of human impedance provides the basis for several theories in human motor control such as the $\alpha$-model and $\lambda$-model equilibrium point theories, the virtual trajectory theory, and dynamic interaction in manipulation, (Hogan 1985). Furthermore, the neuromuscular control system has highly developed selfadaptive properties. The dynamic response of a limb is largely insensitive to external forces in a wide range. It also appears that adaptive compensation for changes of this kind occurs very rapidly in relation to the dominant dynamics of the limb system.

There are a variety of experimental and theoretical techniques developed to model biomechanics and the human motor system. A classic way to characterise a system is done by perturbation analysis, through applying an external perturbation and observing changes in the dynamic of system.

Therefore, to explore this technique and to accomplish studies and experiments in modelling human motor control of the upper limb, it is required to record several variables ranging from biomechanical to bioelectric. Additionally, it also requires some mode to modify or perturb the mechanics

*Corresponding author. Email: afruiz@iai.csic.es

ISSN: $1176-2322$ print / 1754-2103 online 
of movement, in such a way that in literature some studies present manipulators and robotic devices to carry out this task (Mussa-Ivaldi et al., 1985; Scott, 1999; Acosta et al., 2000; de Vlugt et al., 2003).

Keeping the above issues in mind and to encourage studies of this kind, an experimental platform has been designed and developed which integrates several devices and modules to acquire and record information from human upper limb motion and electric activity of muscles. The platform is based on an upper limb robotic exoskeleton that provides a way to apply specific force fields and mechanical perturbations to different human joints. Furthermore, the device can apply force fields to the human joint within its natural workspace, which is not possible with traditional robotic manipulators which impose mechanical constraints.

This paper describes the design, development and validation of the experimental platform and presents the experiments carried out as well as the results obtained using the system to characterise human elbow dynamics. Safety and dependability were milestones in the design phase, according to the guidelines in literature, (Alami et al. 2006). The protocol and experiments were designed to identify and characterise human elbow joint dynamics in terms of mechanical impedance. Moreover, it obtained the EMG activation patterns in the adaptation process under dynamic conditions (torque perturbations).

The next section presents the architecture of the platform and a detailed description of the devices compounding the platform. Section 3 analyses the mathematical background involved in joint dynamics characterising. Section 4 presents simulations of experiments carried out with the platform. Section 5 describes the protocol and experimental methodology used with subjects. Finally, the conclusions and future research are discussed.

\section{Experimental platform}

The experimental platform is composed of modules which provide several capabilities: an upper limb robotic exoskeleton, an EMG module and a control unit. There is a software application to manage the system, set up the experiments, and acquire and store the data. Figure 1 displays the schema of the platform.

Exoskeletons are mechatronic human-centered devices externally coupled to the person (a 'wearable' robot) (Pons 2008). An increasing number of developments have been realised in applications such as tele-operation and power amplification (Schiele and Visentin, 2003), rehabilitation and assistance of disabled or elderly people (Perry and Rosen 2006; Kiguchi and Fukuda 2004; Tsagarakis and Caldwell 2003). It has also been considered to realise experiments and studies on motor control, adaptation and neuro-motor research (Mistry et al. 2005; Ruiz et al. 2006).

Electromyography (EMG) basically consists of the acquisition, record and analysis of the electric activity generated in nervous and muscles, through the utilisation of surface electrodes, thin-wire electrodes or implanted electrodes (De Luca 2003). Measurements extracted from EMG provides a valuable information about the physiology and muscle activation patterns. The information obtained from EMG reflects the forces that will be generated by the muscles and the intervention timing of motor commands. Thus, EMG provides a way to detect the response of the human motor system when controlled movements are executed under external dynamic conditions.

The control of the powered exoskeleton and the data acquisition from the exoskeleton and EMG module was implemented in the MatLab Real-Time suite by MathWorks, Inc. This environment provides mathematical libraries that help implement control strategies in real time and compile them in a C-language executable application to be run on a hardware controller. Thus, the experimental platform has a real-time target computer system (xPC Target) to control the entire system. The following sections describe each module and how they interact.

\subsection{Control unit}

The control unit contains the processing and interfacing elements in the experimental platform. It is composed of a controller to execute the control algorithms and a data acquisition board. The controller is a CPU based in the $\mathrm{PC} / 104$ standard running at $650 \mathrm{MHz}$. The control algorithms are executed in real time, through a kernel installed in the flash memory of the controller. Thus, it allows to run control loops at very high update rates.

The data acquisition board provides channels $\mathrm{A} / \mathrm{D}$ with a resolution of 16 bits. It also provides channels $\mathrm{D} / \mathrm{A}$ with 12 bits. The sensors of the exoskeleton and the conditioned signals of the EMG module were connected to this data acquisition board. Additionally, the control unit provides a TCP/IP ethernet interface to communicate with the computer program installed in a remote host.

\subsection{Upper-limb robotic exoskeleton}

Wearable Orthosis for Tremor Assessment and Suppression (WOTAS) is a powered exoskeleton designed for the upper limb spanning elbow and wrist joints. It was implemented for functional compensation of handicapped people with movement disorders such as tremor (Rocon et al., 2007). This system was an open platform to test nongrounded tremor reduction strategies by applying biomechanical loading on tremorous movement.

The robotic device provides a way of manipulating the mechanical conditions of each joint independently since the mechanical loads are applied by the exoskeleton directly to the arm and forearm (see Figure 2), (Ruiz et al. 2006). Elbow flexo-extension, wrist flexo-extension and forearm 


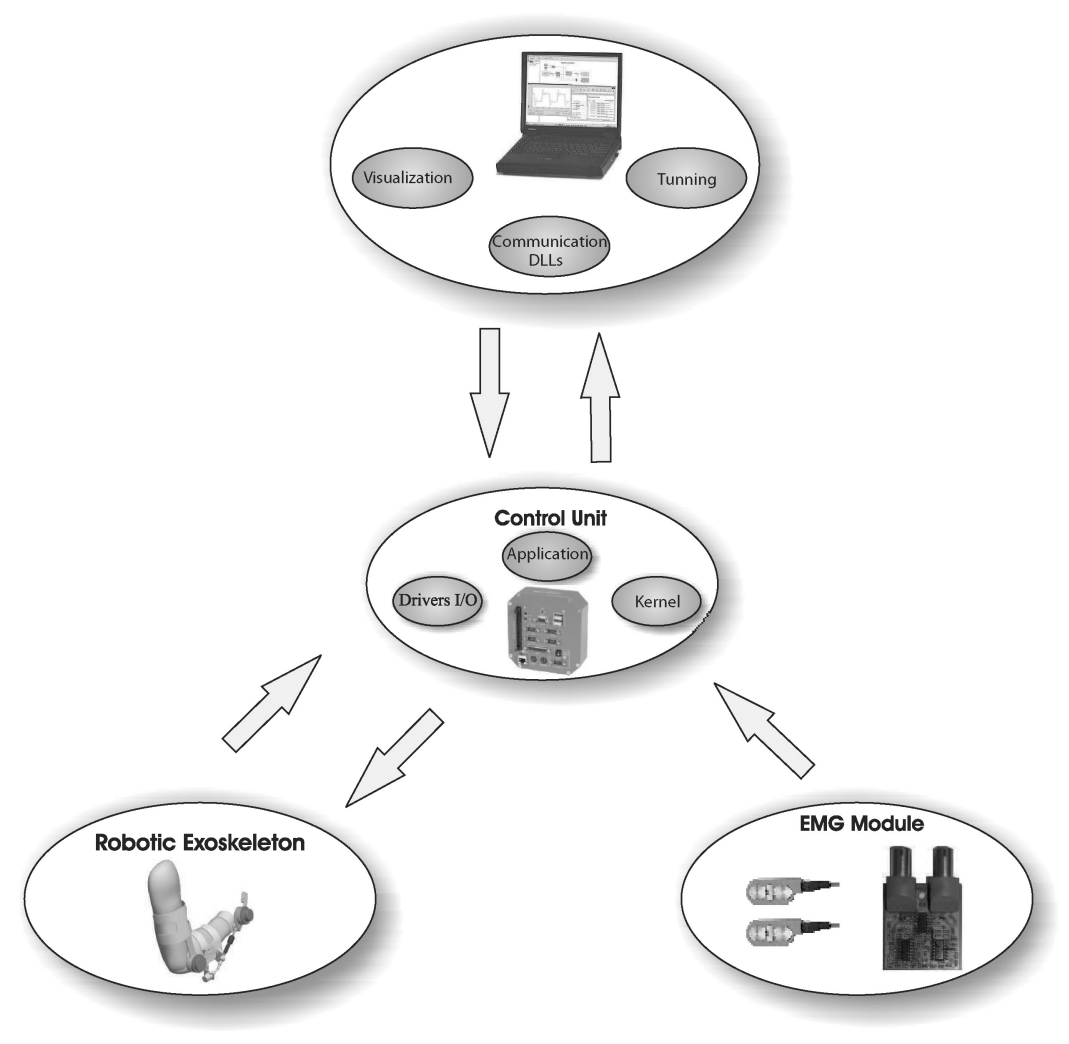

Figure 1. Architecture of the exoskeleton-based experimental platform.

prono-supination are the arm movements which may be modified.

Sensors on the powered exoskeleton measure the biomechanics of the arm (wrist and elbow) that allow to define a number of limb movements, motor tasks and several postures under various mechanical conditions. It is equipped with kinematics sensors for angular position and velocity using precision potentiometers and gyroscopes, respectively. It is also equipped with kinetic (interaction force between limb and device) sensors using strain gauges. The actuation of the robotic device was implemented using DC flat brushless motors.

\subsubsection{Control strategies}

The control of the exoskeleton was implemented through impedance control, and its schema is illustrated in Figure 3. Impedance control is a strategy for constrained motion rather than a concrete control scheme. The basic idea of this approach is to have a closed-loop control system whose dynamics can be mathematically described by Equation (1), (Hogan 1985):

$$
F=I\left(\ddot{q}-\ddot{q}_{0}\right)+B\left(\dot{q}-\dot{q}_{0}\right)+K\left(q-q_{0}\right),
$$

where $I, B, K$ represent the inertia, damping and stiffness of the interactive system, respectively.

Inertia, damping and stiffness can be adjusted by the control system according to the goals pursued (impedance may vary in the different task space directions, typically in a non-linear and coupled way). The objective of the control was to modify the apparent impedance that the exoskeleton provides to the human arm. The interaction between the robot and a human then produces a dynamic balance between these two 'systems'.

Control is based on a dual control loop (3). The value of torque applied by the exoskeleton to the upper limb, $\tau_{d}$, is calculated on the basis of the combined effects of both loops:

$$
\begin{aligned}
\tau_{d} & =f_{d}-f_{m t}-\tau \\
& =f_{d t}-I \cdot \ddot{q}_{t}-B \cdot \dot{q}_{t}-K \cdot q-\tau .
\end{aligned}
$$

This closed-loop control schema uses information from gyroscopes $(\dot{q})$ and potentiometers $(q)$. This information defines the actual impedance force, $f m_{t}$, after multiplying them by coefficients. The lower loop of control schema serves the goal of minimising the effect of the exoskeleton on voluntary motion. In this case, force sensors measure the interaction force between the exoskeleton and upper 


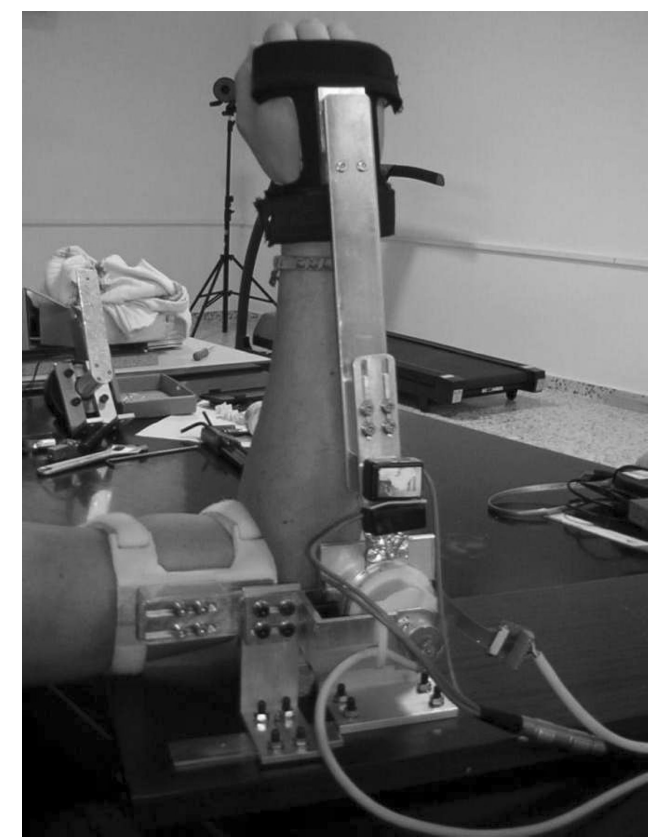

Figure 2. Upper-limb robotic exoskeleton aimed at human motor control research.

limb, $f$. In order to achieve this, the interaction force, $f$, is filtered so that only the force opposing voluntary motion, $\tau$, is fed back into the lower branch of the control loop.

\subsubsection{Safety issues}

Several redundant safety systems have been implemented for the robotic device. The segments of the exoskeleton are constrained to move within the natural motion range of upper limb. In addition to this physical stop, the experimenter holds a switch that may be pressed during an experiment to interrupt the power supply to exoskeleton actuators. Safety concerns in algorithms have also been implemented, limiting the current provided to the actuators if it reached the maximum velocity in a specific slice time.

\subsection{EMG acquisition module}

The EMG acquisition module in the experimental platform permits to acquire data of several muscular groups and is directly interfaced to the control unit. Since the EMG signal is very small $(50 \mu V \sim 5 m V)$, the acquired signal may be affected by interference from other biological and environmental sources like movement artifacts, electric noise, muscle noise, etc. Thus, the raw EMG signal was amplified and filtered to be acquired in the data acquisition board. The electronic circuit developed provides amplification gains between 300 and 2400 and has a pass-band filter with a cut-off frequency between 20 and $500 \mathrm{~Hz}$.

To pick up the EMG signal, surface electrodes were used because they are noninvasive and are easy to use. To avoid the detection of other electrical signals in the proximity of the detection surface except signals of interest, bipolar electrodes were used. A great challenge to the accuracy of the system is the presence of EMG electrodes attached to the skin, because during the movement an unaccounted motion exists between the skin and the bones. Furthermore, the robotic exoskeleton coupled to the upper limb modifies the electrode-skin impedance.

Additionally, a battery was used to power the EMG acquisition module in order to reduce $50 \mathrm{~Hz}$ hum (powerline noise) from the EMG signal acquired.

\subsubsection{Safety issues}

Taking into account international safety regulations about of electronic devices which are connected to a person, several topics were addressed for electric isolation of the EMG module. It is not convenient to make an electrical connection between a person and equipment powered through electrical wiring because in the case of a failure in the equipment, the subject may be exposed to deadly voltages. Furthermore, the patient is parasitically coupled to various sources of noise such as power supplies and fluorescence lamps. Thus, the parasite voltages induced in the body may

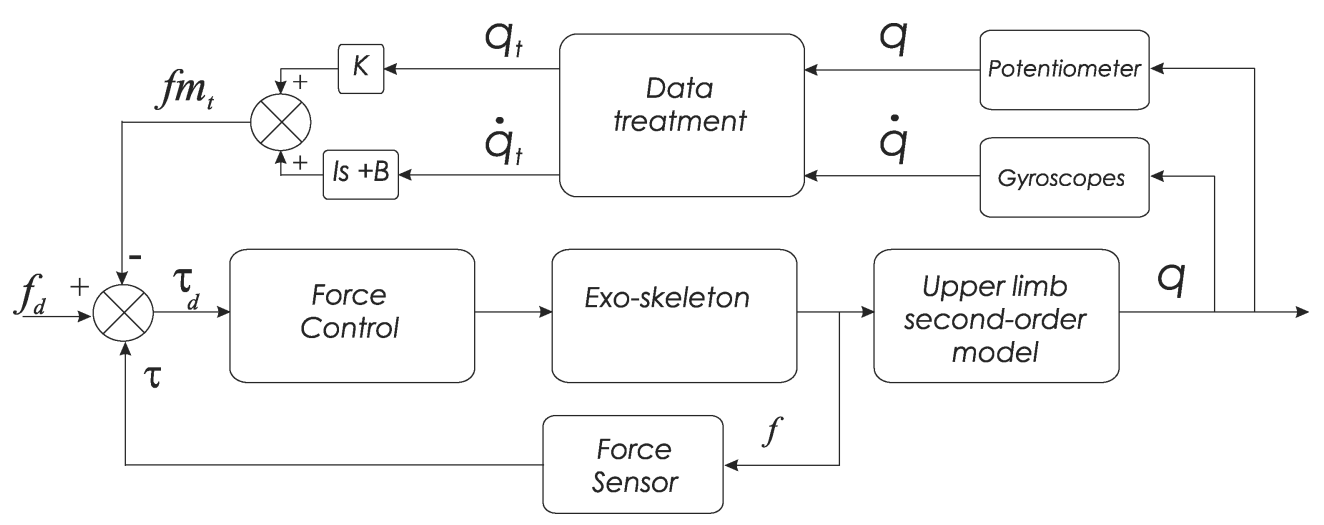

Figure 3. Impedance-based control strategy implemented in the robotic exoskeleton. 
generate leakage currents from the body, through the module to ground.

The electric isolation of the subject from ground has been implemented using a wide-band, unity-gain isolation amplifier. The selected amplifier (Burr Brown ISO-124) has the input and output sections isolated from each other and provides isolation of electrical signal through capacitive coupling.

\subsection{Host computer}

In order to provide an interface to manage the entire system (see Figure 1) a software application was developed. It communicates with the control unit (by Ethernet TPC/IP) using Dynamic Link Libraries (DLLs). The control algorithms are downloaded and its execution controlled from the software application. The user interface runs on a Windows machine and provides capabilities for data acquisition, recording, analysing and graphically displaying. Moreover, it permits to tune the parameters that require adjustments in the experimental platform.

\section{Neural control of the human upper limb and joints}

The human modulates their motor patterns in order to compensate for different sorts of loads found in a wide range of contexts. Each object or environment creates forces with different space and timing characteristics such as gravitational forces, viscous forces and accelerative forces (Scott, 2005). The human is able to compensate for such dynamic environments.

Thus, there is a basic compensation mechanism that exploits the visco-elastic properties of the neuromuscular system. Control of the impedance of the neuromuscular system is a form of adaptive behaviour that the central nervous system (CNS) uses to accommodate perturbations from the environment (Hogan 1984). The CNS avoids the kind of complex computation involved in motor control through the visco-elastic properties of the neuromuscular system, the muscles and the reflex loops. This visco-elasticity is able to generate restitution forces against external perturbations and may be considered as a control gain in the peripheral feedback.

Under external perturbations, the intrinsic mechanical response of muscle is immediate. On the other hand, reflex responses and other responses that act by way of changes in muscle activation can only produce force after some delay with respect to the movements that trigger them, (van der Helm et al., 2002). Intrinsic muscle visco-elastic forces are in phase with the movements that produce them.

Although intrinsic stiffness is directly proportional to joint torque, it is possible to vary total joint stiffness and damping independently of joint torque. Theoretically, this can be done by
(1) varying the level of co-contraction of antagonist muscles;

(2) varying the reflex gains for velocity feedback and position feedback independently of joint torque.

\subsection{Mathematical models}

In the human motor control field, to quantify joint stiffness, viscosity and inertia, small-amplitude random perturbations (zero mean) can be applied about the flexo-extension rotation axis at specific muscle contraction levels (see Figure 4).

The relation between small displacements $(X)$ of a degree of freedom of a musculoskeletal system and the external forces $(F)$ associated with such displacements can be represented by a linear transfer function which is usually named the mechanical impedance $(F=X)$ or admittance $(X=F)$. Using a simple linear control system, it is explained here how the components of the control system influence the mechanical impedance.

The model is non-linear and varies depending on factors such as torque bias and posture. Thus, in order to fit the data to a second-order linear model, an operating point must be specified. The operating point consists of constant posture, constant force and non-fatiguing contractions for a particular task. Parameters in the model change as the operating point changes. The linear model can be defined by the linear Equation (3).

$$
\tau(t)=M \frac{\partial^{2} \theta(t)}{\partial^{2} t}+B(\delta) \frac{\partial \theta(t)}{\partial t}+K(\delta) \theta(t)
$$

where $\delta$ defines the operating point of the system. Equation (3) is useless when the operating point varies in a considerable way, (Kearney and Hunter 1990).

In literature, during an isometric contraction in which a subject voluntarily increases or decreases the joint torque by changing muscle activation, joint stiffness has been shown

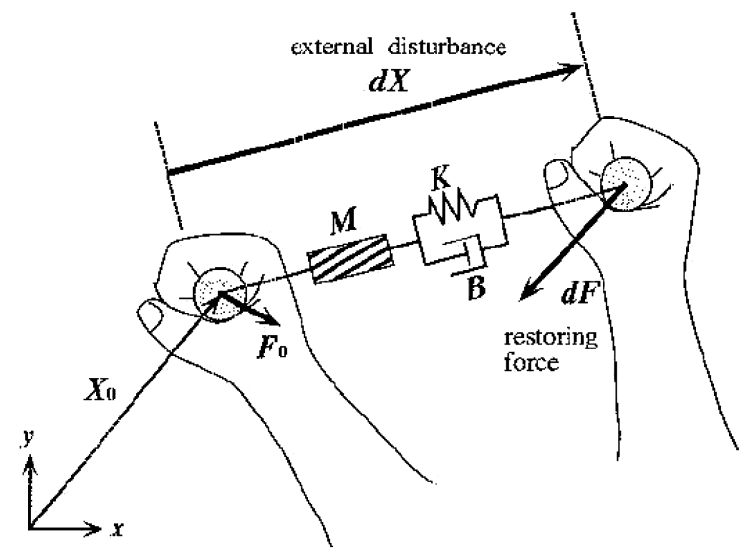

Figure 4. Mechanical perturbation for system identification of the elbow joint dynamics. 
to increase in parallel with joint torque. Reflex gain also increases in parallel with muscle activation provided that the contraction is well below maximal. Furthermore, for experimental conditions that involve quasi-static and postural tasks, parameters are a function of the EMG amplitude estimations $\left(\hat{s}_{E}, \hat{s}_{F}\right)$. A linear second-order equation that relates EMG amplitude and angular displacement with changes of generated torque in joint, can be modelled as

$$
\Delta \tau=I \cdot \Delta \ddot{\theta}+B\left(\hat{s}_{E}, \hat{s}_{F}\right) \cdot \Delta \dot{\theta}+K\left(\hat{s}_{E}, \hat{s}_{F}\right) \cdot \Delta \theta,
$$

where $\hat{s}_{E}$ is the estimation of the EMG amplitude of extensor muscles, $\hat{s}_{F}$ is the estimation of the EMG amplitude of flexor muscles, $\Delta \theta$ is the variation of angular displacement in the joint, $\Delta \tau$ is the variation of generated torque in the joint, $K\left(\hat{s}_{E}, \hat{s}_{F}\right)$ is the stiffness function, $B\left(\hat{s}_{E}, \hat{s}_{F}\right)$ is the viscosity function and $I$ is the inertial parameter.

\subsection{Identification system methods}

Elbow joint stiffness $K$, viscosity $B$, and inertia $I$ were identified from the measured angular joint displacement $\Delta \theta$ and external applied torque $\tau(t)$. Gravitational torque was assumed constant under the small-amplitude perturbation and thus would not appear in the relationship characterising elbow dynamics in terms of $\mathrm{K}, \mathrm{B}$ and $\mathrm{I}$.

To accomplish the system identification, the ARX model has been used to estimate parameters of the elbow mechanical impedance. The structure of ARX model is represented as a transfer function, such as shown in Equation (5), (Ljung 1999)

$$
\frac{Y(q)}{U(q)}=q^{-n k} \frac{B(q)}{A(q)} .
$$

In Equation (5), $Y(q)$ and $U(q)$ represent input and output data, respectively. $A(q)$ and $B(q)$ are polynomials in the delay operator $q^{-1} . A(q)$ and $B(q)$ are defined as

$$
\begin{aligned}
& A(q)=1+a_{1} q^{-1}+\ldots+a_{n a} q^{-n a} \\
& B(q)=b_{1}+b_{2} q^{-1}+\ldots+b_{n b} q^{-n b+1},
\end{aligned}
$$

where $n a$ and $n b$ are the order of polynomials $A(q)$ y $B(q)$ respectively, while $n k$ is the sample number between input and output. Replacing the expressions of $A(q)$ and $B(q)$ from Equations (6a) and (6b) in (5), is obtained

$$
A(q) y(t)=B(q) u(t-n k) .
$$

Relationship between input and output data can be represented by a linear difference equation (replacing (6a) and (6b) in equation (7))

$$
\begin{aligned}
& y(t)+a_{1} y(t-1)+\ldots+a_{n a} y(t-n a) \\
& \quad=b_{1} u(t-n k)+\ldots+b_{n b} u(t-n k-n b+1) .
\end{aligned}
$$

Equation 8 relates actual output $y(t)$ to a finite number of past outputs $y(t \ldots k)$ and actual inputs. To identify the system, the present work utilises the applied torque $\tau(t)$ as input, and the angular displacement $\theta(t)$ as output.

\section{Simulations methods}

The visco-elastic properties of the human arm and joints vary in a wide range, (Mussa-Ivaldi et al., 1985; Zhang and Rymer 1997). However, experiments in the present study span particular operating conditions for elbow joint (postural, isometric) which impedance parameters should not vary steeply.

Analysis and simulations were made for the conditions similar to those for the human arm experiments. A set of known values for elbow joint dynamics, reported in literature, were used in simulations (Zhang and Rymer 1997). Simulations permit to validate processing techniques for the measured signals and to determine signal-to-noise ratio (SNR) limits for a reliable estimation.

The torque perturbation was the stimulus for the secondorder system (Equation (3)) and was simulated using a pseudo-random white noise generator followed by a low-pass Butterworth filter with a cut-off frequency of $10 \mathrm{~Hz}$.

Impedance parameter estimation consists of estimating $\mathrm{K}, \mathrm{B}$ and I given $\Delta \tau$ and $\Delta \theta$ using the parametric model in Equation (3). It was simulated noise in signals with a noise power spectral density in several magnitudes. The estimated and known values were compared, using the RMS error index. RMS error (root-mean-square) was defined for real and estimated values, such as Equations (9a) and (9b).

$$
\begin{aligned}
& \text { error RMS in } \boldsymbol{K}=\sqrt{\frac{\sum_{i=1}^{N}\left(K_{\text {real }}-K_{\text {estimated }}\right)^{2}}{N}} \\
& \text { error } R M S \text { in } \boldsymbol{B}=\sqrt{\frac{\sum_{i=1}^{N}\left(B_{\text {real }}-B_{\text {estimated }}\right)^{2}}{N}},
\end{aligned}
$$

where $K_{\text {real }}$ and $B_{\text {real }}$ represent the simulated (real) values and $K_{\text {estimated }}$ and $B_{\text {estimated }}$ represent the estimated values. $\mathrm{N}$ is the number of simulated samples.

To determine the SNR limits values for biomechanical and electromiographic signals, it was defined a relative error of maximum $10 \%$ in the estimation process. Thus, to obtain biomechanical signals it should have $S N R>15$ and EMG signals should have $S N R>18$. The values of SNR impose a lower limit to the signals for a reliable estimation.

Biological systems such as the human motor system are characterised as time-varying systems. Thus, in order to assess the validity of the parameters estimation procedures in systems with time-varying parameters, a set of simulations 
were carried out. Maximum frequencies of the parameters were obtained in order to obtain acceptable errors (relative error $<10 \%$ ).

$B$ and $K$ parameters (Equation (3)) were tuned to vary as sinusoidal functions. In simulations, the sinusoidal frequency was increased until it was calculated as the maximum estimation error. It obtained a maximum frequency of $0.7 \mathrm{~Hz}$, in order to estimate reliable parameters. Figure 5 shows real and estimated values for stiffness and viscosity.

The experimental protocol with real subjects involves subjects executing isometric tasks, with constant cocontraction. Such postural tasks exhibit torque variations, i.e. torque bias. In literature, bias is low-frequency, lower that the obtained limit in simulations $(0.7 \mathrm{~Hz})$.

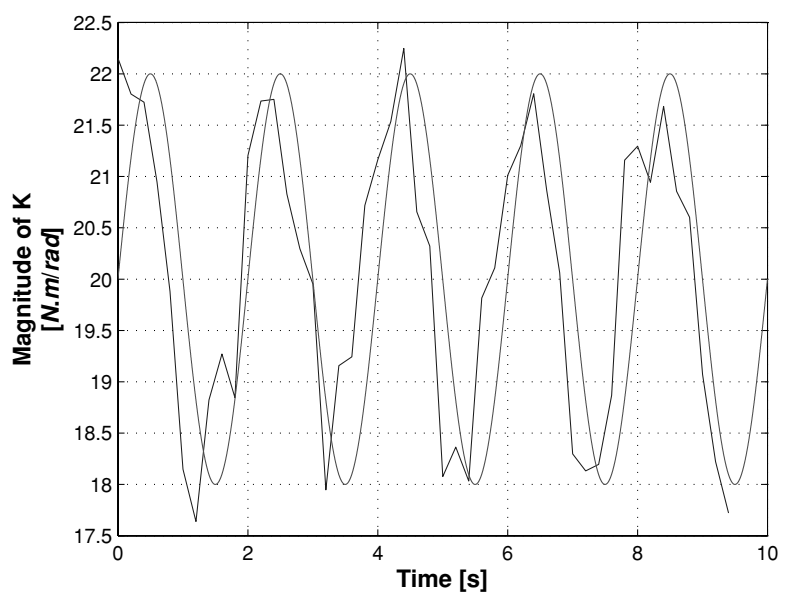

(a) Estimation of time-varying stiffness.

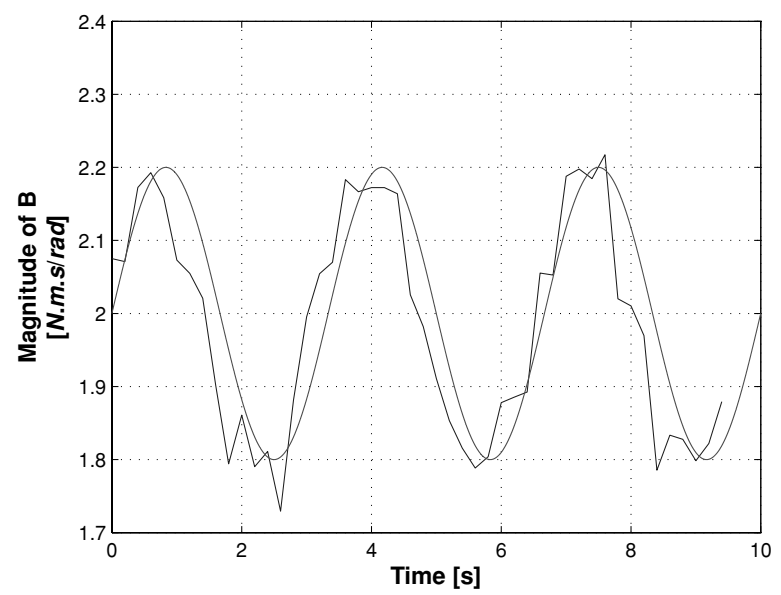

(b) Estimation of time-varying viscosity.

Figure 5. Estimation of time-varying parameters of $\mathrm{K}$ (upper) and B (lower). Both parameters were tuned to follow sinusoidal functions. Real values are in red, estimated values in blue. This figure can be seen in colour online.

\section{Experimental methods}

To characterise the dynamic behaviour of the elbow joint during postural control, mechanical torques were applied by the powered exoskeleton at the forearm segment (see Figure 2). The angular displacement of the elbow away from the maintained posture and the applied torque were used in an identification system process. In order to correlate dynamic behaviour of the elbow joint with bioelectric information, it recorded muscle activation patterns of implied muscles around this joint. It validated a preliminary linear approximation between visco-elasticity and EMG.

\subsection{Validation trials}

The torque profiles were validated experimentally with one healthy subject. The robotic device was configured to apply resistance dependent on position (spring-like load), velocity (viscosity) and acceleration (inertial load). Thus, the torques (loads) indicated by the vector $\tau$, were computed as a function of the angular kinematics in the human elbow, according to the following equations

$$
\begin{aligned}
\tau_{1} & =K \cdot\left(\theta(t)-\theta_{0}\right) \\
\tau_{2} & =B \cdot \dot{\theta}(t) \\
\tau_{3} & =I \cdot \ddot{\theta}(t),
\end{aligned}
$$

where $\theta(t), \dot{\theta}(t)$ and $\ddot{\theta}(t)$ are the human elbow angular displacement, velocity and acceleration vectors, respectively; $\theta_{0}$ is an equilibrium point of the angular position; and $\mathrm{K}, \mathrm{B}$ and I are constant vectors representing spring-like, viscous and inertial loads, respectively, of the imposed environment at the elbow level.

Rhythmic movements at the elbow level were executed by a subject wearing the exoskeleton. In each trial a resistance was delivered according to the above equations. Figure 6 presents the experimental relationship between the torque and its corresponding kinematic parameter.

The small phase-lag observed in the Figure 6 for each delivered load and its kinematic variable was due to the low-pass filters in the control algorithms and the filtering imposed by the human upper limb (muscle, soft tissues, etc). Thus, inconsistencies between the measured and predicted moments can be attributed to a variety of factors, such as the placement of the electrodes or the contribution of the passive structures at the joint. In the future there may be the need to better account for the contribution of passive structures at the joint, while maintaining the simplicity of the model (Figure 7).

\subsection{Protocol}

Four healthy adult volunteers (mean age $28 \pm 3$, mean height $1.73 \pm 0.04 \mathrm{~m}$ ) were instrumented with surface 


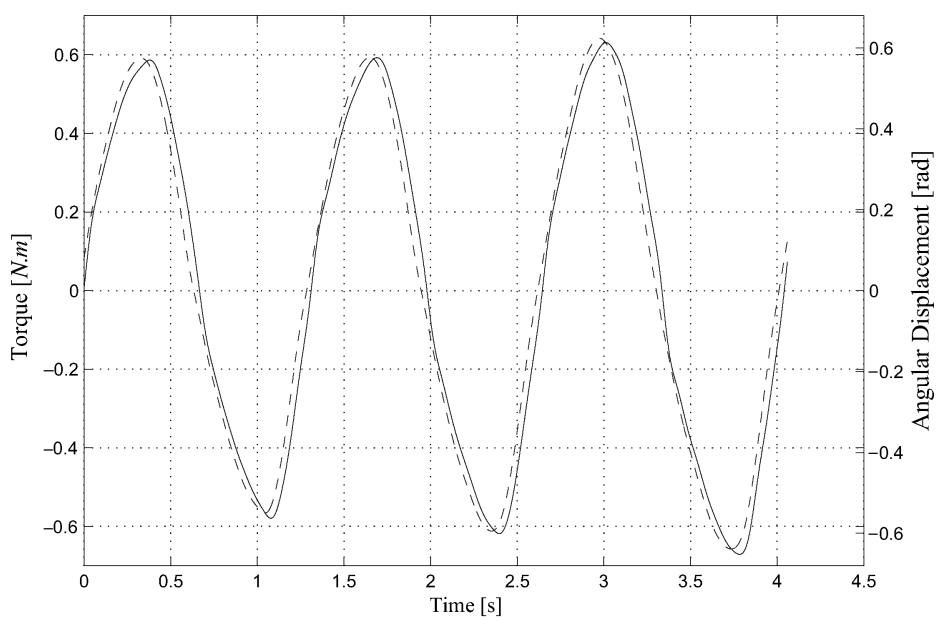

(a) Torque $\tau_{1}$ generated by equation 10a. Magnitude of $\mathrm{K}$ of 1 N.m/rad.

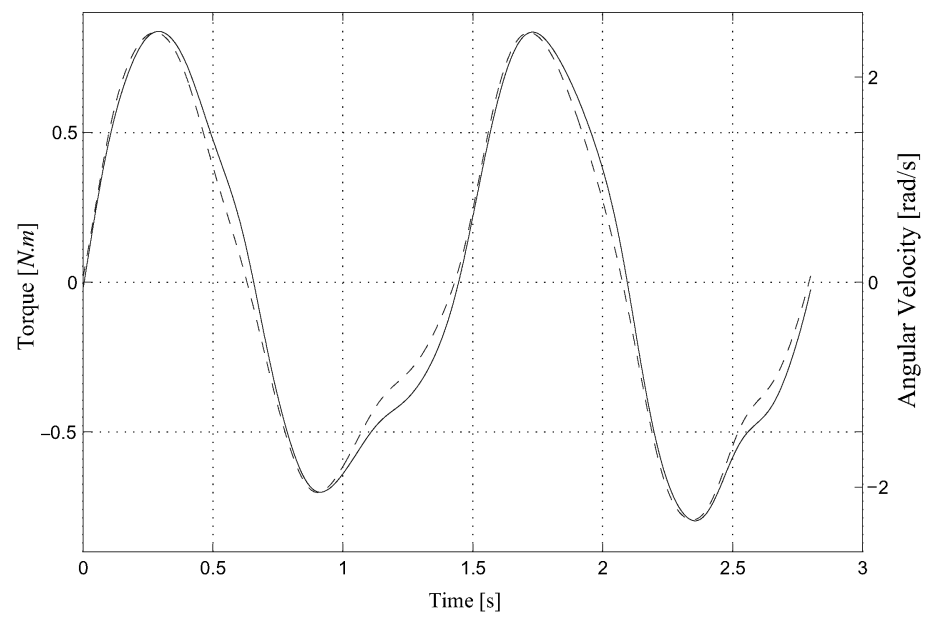

(b) Torque $\tau_{2}$ generated by equation $10 \mathrm{~b}$. Magnitude of $\mathrm{B}$ of 0.3 N.m.s/rad.

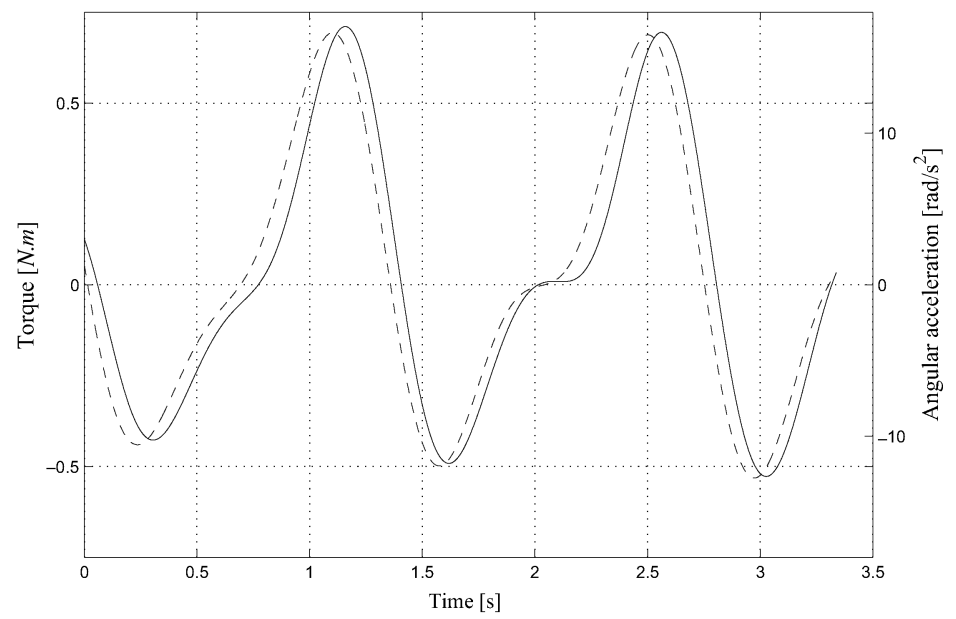

(c) Torque $\tau_{3}$ generated by equation 10c. Magnitude of I of 0.04 N.m.s ${ }^{2} / \mathrm{rad}$.

Figure 6. Torque profiles applied by exoskeleton according to: (a) angular displacement, (b) velocity and (c) acceleration. 


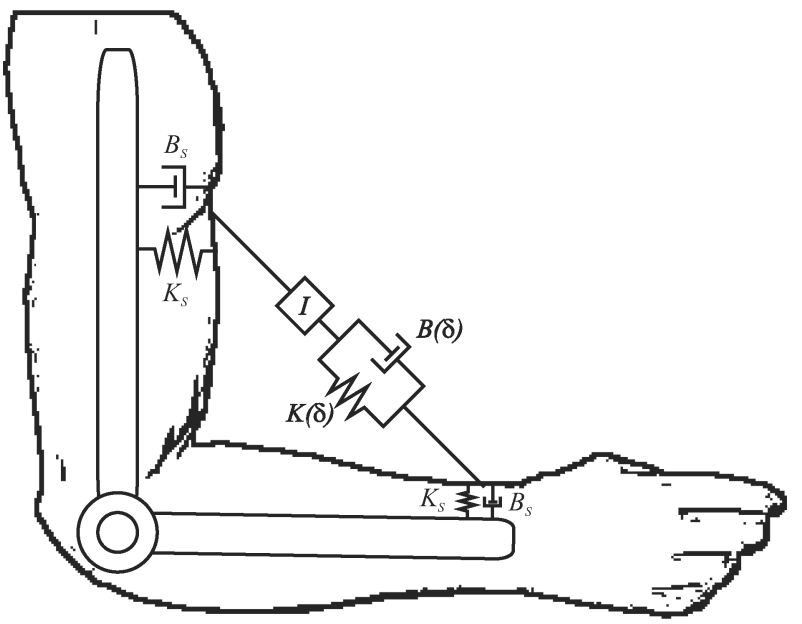

Figure 7. Passive structures and dynamics involved in human upper-limb.

EMG electrodes following the SENIAM recommendations. Two upper arm muscles were measured: the flexor (biceps brachii) and extensor (triceps brachii long head) muscles involved in the elbow joint movement.

Subjects wore a robotic exoskeleton on the right arm allowing elbow flexion and extension in the vertical plane. The shaft joint on the device was aligned with the subject's elbow joint, and the device was attached to the upper arm and forearm (see Figure 2).

The experiments consist of static (isometric, constant force) contractions of the muscles and maintaining posture. At one specific time instant, the exoskeleton applied unexpectedly random torque perturbations on the human forearm. Subjects were instructed to maintain the forearm posture while resisting external perturbations. In this case the elbow joint does not move, i.e. a fixed position is being maintained, then the setpoint of the velocity feedback is zero and the setpoint of the position feedback is the equilibrium position. The feedback damping and stiffness will act to restore the joint to this state. Thus, visco-elastic parameters were estimated by applying perturbations about the equilibrium position.

Additionally, subjects were asked to maintain a steady background muscle activation throughout the trial. Five repetitions were chosen for each experimental session and data were sampled at $1 \mathrm{kHz}$ for biomechanical variables (kinetics and kinematics) and for the EMGs.

\section{Experimental results}

\subsection{Data analysis}

The signal-to-noise ratios were calculated for all signals in order to validate them according to results obtained in simulations. The kinematics and kinetics data have been filtered through a four-pole Butterworth filter with a cut-off frequency of $10 \mathrm{~Hz}$. Surface EMG signals were rectified and the envelope of the EMG signals extracted using a low pass filter with a cut-off frequency of $10 \mathrm{~Hz}$. It used a 5 th order Butterworth filter for this propose. EMG amplitude estimation was accomplished using its RMS magnitude

$$
R M S=\sqrt{\frac{1}{n} \sum_{i=1}^{n} x_{i}^{2}}
$$

where $x_{i}$ is the voltage value in the $i$-esima sample and $n$ is the number of samples of the segment.

The recorded data set has been subdivided into smaller data sets. Moreover, individual 'windows' overlap in time, such as shown in Figure 8. Whole data sets were separated in windows $\left(S_{1}, S_{2} \ldots S_{n}\right)$ of N-samples length. For each window, the elbow joint parameters, i.e. the mechanical impedance were estimated. Windows were overlapped to identify time trends and variations of impedance parameters.

\subsection{Results}

To evaluate joint visco-elasticity during the postural experiments, previous studies which had suggested a linear relation between surface EMG activity and joint torque and joint stiffness was used, (Osu and Gomi 1999; Osu et al. 2004). They defined a parameter called index of muscle cocontraction around the joint (IMCJ). The IMCJ was defined as the summation of the absolute values of agonistic and antagonistic muscle torques around the joint and computed from the linear relation between surface EMG and joint torque.

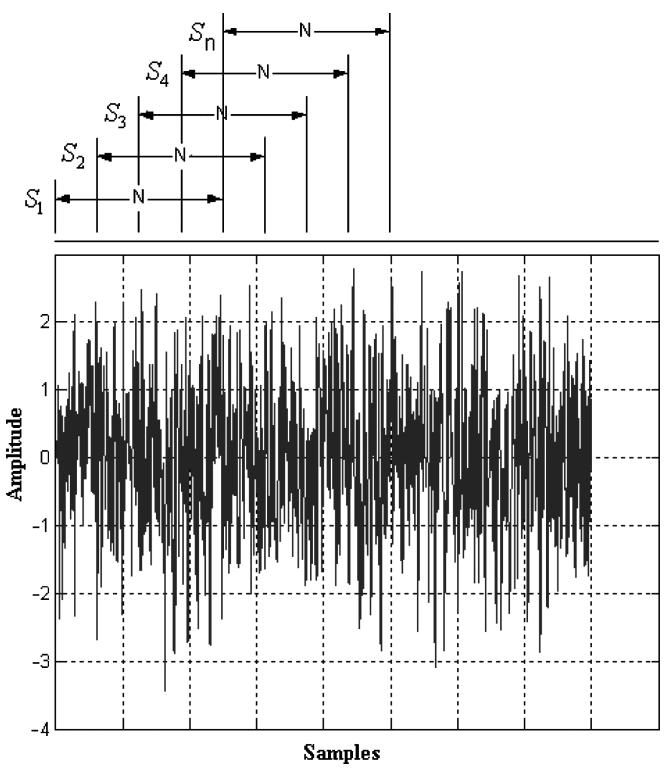

Figure 8. Overlapping of recorded data set. 


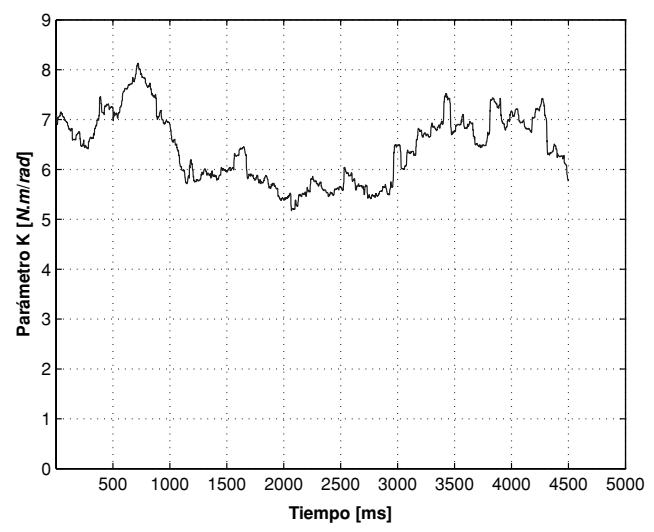

(a) Estimation of viscous parameter $B$.

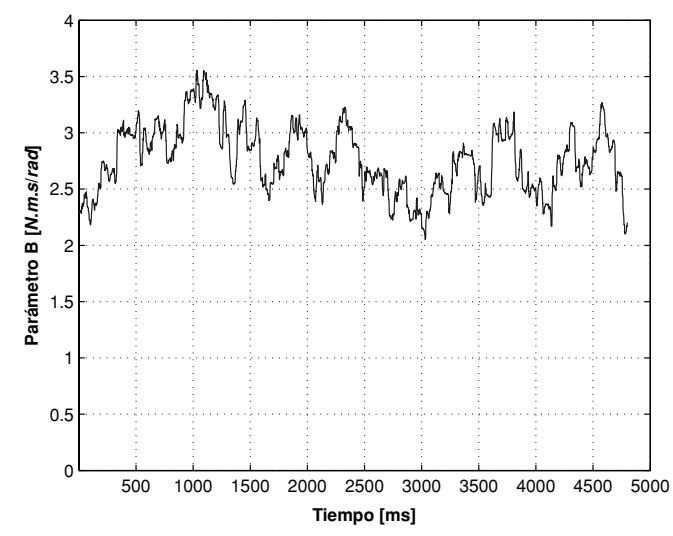

(b) Estimation of stiffness $K$.

Figure 9. Estimated values using recorded data for visco-elasticity of elbow joint.

IMCJ around elbow joint can be expressed as the equation below, (Osu and Gomi 1999; Osu et al. 2004). $s_{F 1}$ and $s_{E 1}$ are the surface EMG activity of the elbow monoarticular flexor and extensor, respectively, and $s_{F 2}$ and $s_{E 2}$ denote surface EMG activity of bi-articular flexor and extensor, respectively.

$$
I M C J=a_{1} s_{F 1}+a_{2} s_{E 1}+a_{3} s_{F 2}+a_{4} s_{E 2} .
$$

In literature, human arm visco-elastic parameters are a function of muscular activation. For experimental conditions that involve isometric, quasi-static and postural tasks, it validates the stiffness and viscosity parameters modelled as linear functions of the EMG amplitude estimations $\left(s_{E}, s_{F}\right)$. Thus, as presented in Equation (4), there is a second-order relationship involving EMG information in the visco-elastic parameters.

Starting from Equation (4), $\Delta \tau, \Delta \theta$ were used to estimate $\mathrm{K}, \mathrm{B}$ and I. Next, the EMG amplitude was calculated

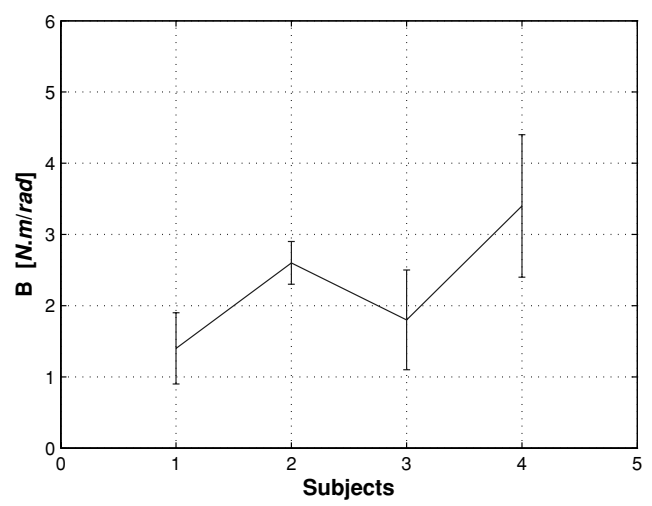

(a) Parameter of viscosity $B$. as well as $\mathrm{K}$ and $\mathrm{B}$ values to correlate the information, using linear least squares.

Figure 9 presents the estimation of $K$ and $B$ respectively, for one subject. Such parameters were obtained after calculating individual parameters of the overlapped windows.

In previous Figures, there are approximately constant parameters. Variations arise from the torque modulation generated by subjects in order to maintain the postural task. Magnitudes in the range of 2.5 a 4.7 N.m.s/rad for viscosity and 5.3 a $13.1 \mathrm{~N} . \mathrm{m} / \mathrm{rad}$ for stiffness parameters obtained. Those values in the range found in literature.

Figure 10 shows mean and standard deviation in parameters $B$ and $K$ for subjects. For each subject (x-axis), the average of recorded values was calculated in all repetitions.

\section{Conclusions and future work}

An experimental platform has been developed to set up experiments in human movement and neuro-motor control,

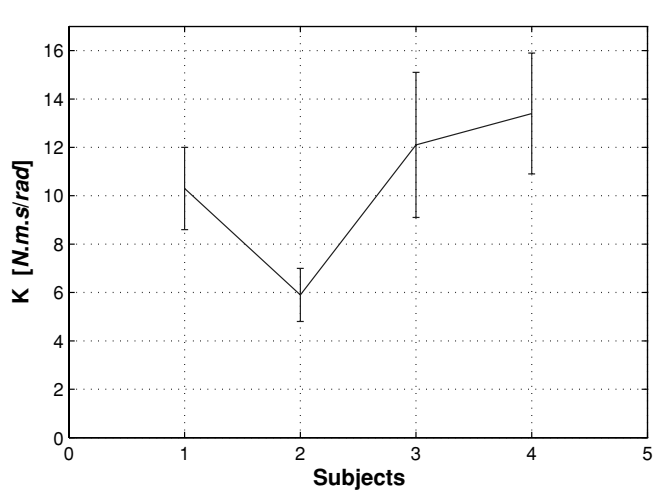

(b) Parameter of stiffness $K$.

Figure 10. Mean and standard deviation in estimated parameters. X-axis represents each subject. 
integrating an upper limb robotic exoskeleton as well as a tool to monitor the bioelectric muscular activity of the subject. The platform enables studies in biomechanics, modelling and analysing human motion in such a way that may simultaneously record sensory and motor responses during specific motor tasks.

The mechanical impedance of a system is best described by its transfer function, which can only be estimated using perturbations. However, such perturbations interact with the natural behaviour of the motor control system. In the present study, the neuromuscular system was approximated with a second-order model. The elbow joint system is higher order and one might therefore question the accuracy of the obtained stiffness and damping estimates. However, the goal was to obtain the modulation and prediction of stiffness and damping using EMG data and not in their exact values.

Simulation results provide information about the required quality of the recorded signals. Thus, a SNR minimum of 18 for biomechanical signals and SNR minimum of 15 for EMG signals, in order to have a reliable estimation of parameters (with an error $<10 \%$ ).

Afterwards, the dynamics of human elbow joint was estimated in postural control. An outstanding result was correlating visco-elasticity and EMG data. A linear relationship was defined between stiffness as well as viscosity and the EMG amplitude estimation. The obtained values were in the range found in literature.

In order to obtain a more accurate model of the joint dynamics, it is required to include the non-linearities and reflexes involved (soft tissues, muscular spindles, etc.). However, the compact and simple model presented in this study is enough to characterise the main factors relating articular dynamics with muscular activation.

Obtained results in this work provide information to the field of human motor control and also to its application in robotics and other engineering applications. Thus, through the characterisation of mechanical properties associated with the human joints as well as strategies used by human beings to command it, exist a direct application to control devices based on EMG in a biologically inspired way, i.e. biomimetic control.

\section{References}

Acosta AM, Kirsch RF, Perreault EJ. 2000. A robotic manipulator for the characterization of two-dimensional dynamic stiffness using stochastic displacement perturbations. J Neurosci Meth. 102(2):177-186.

Alami R, Albu-Schaeffer A, Bicchi A, Bischoff R, Chatila R, de Luca A, de Santis A, Giralt G, Guiochet G, Hirzinger G, Ingrand F, Lippiello V, Mattone R, Powell D, Sen S, Siciliano B, Tonietti G, Villaniet L. 2006. Safe and Dependable Physical Human-Robot Interaction in Anthropic Domains: State of the Art and Challenges. Proc. IROS' 06 Workshop on pHRI - Physical Human-Robot Interaction in Anthropic Domains, Beijing, China. de Luca G. 2003. Fundamental Concepts in EMG Signal Acquisition, DelSys Inc.

Dolan JM, Friedman MB, Nagurka ML. 1993. Dynamic and Loaded Impedance Components in the Maintenance of Human Arm Posture. IEEE Transactions on Systems, Man and Cybernetics, 23(3):698-709.

Herr H, Whiteley GP, Childress D. 2003. Chapter 5: Cyborg Technology - Biomimetic Orthotic and Prosthetic Technology In Biologically Inspired Intelligent Robots. Bar-Cohen, Y. and C. Breazeal, Eds., SPIE Press, Bellingham, Washington.

Hogan N. 1984. Adaptive Control of Mechanical Impedance by Coactivation of Antagonist Muscles. IEEE Transactions on Automatic Control, 29(8):681-690.

Hogan N. 1985. Impedance conrol: An approach to manipulation: Part I-Theory, Part II-Implementation, Part III - Applications. Journal of Dynamics Systems, Measurement and Control, 107:1-24.

Kazerooni H. 1990. Human-Robot Interaction via the Transfer of Power and Information Signals. IEEE Transaction On Systems, Man and Cybernetics, 20(2):450-463.

Kearney RE, Hunter IW. 1990. System identification of human joint dynamics. Critical Reviews on Biomedical Engineering, 18(1):55-87.

Kiguchi K, Fukuda T. 2004. A 3DOF Exoskeleton for Upper-Limb Motion Assist - Consideration of the Effect of Bi-Articular Muscles. Proceedings of the IEEE International Conference on Robotics and Automation.

Kleissen RFM, Buurke JH, Harlaar J, Zilvold G. 1998. Electromyography in the Biomechanical analysis of human movement and its clinical application. Gait and Posture, 8(2):143158.

Kutch JJ, Buchanan TS. 2001. Human elbow joint torque is linearly encoded in electromyographic signals from multiple muscles. Neurosci. Lett. 311(2):97-100.

Ljung L. 1999. System Identification: theory for the user. PrentiveHall, Engelwood Cliffs, 2nd.

Mistry M, Mohajerian P, Schaal S. 2005. Arm Movement Experiments with Joint Space Force Fields using an Exoskeleton Robot. Proceedings of the IEEE International Conference on Rehabilitation Robotics.

Mussa-Ivaldi FA, Hogan N, Bizzi E. 1985. Neural, Mechanical, and Geometric Factors Subserving Arm Posture in Humans. J Neurosc. 5(10):2732-2743.

Osu R, Gomi H. 1999. Multijoint Muscle Regulation Mechanisms Examined by Measured Human Arm Stiffness and EMG Signals. The Am. Phy. Society, 81(4):1458-1468.

Osu R, Kamimura N, Iwasaki H, Nakano E, Harris CM, Wada Y, Kawato M. 2004. Optimal impedance control for task achievement in the presence of signal-dependent noise. J Neuro. 92(2):1199-1215.

Perry JC, Rosen J. 2006. Design of a 7 Degree-of-freedom upper limb powered exoskeleton. Proceedings of the IEEE International Conference on Biomedical Robotics and Biomechatronics (BioRob).

Pons JL. 2008. Wearable Robots: Biomechatronic Exoskeletons. Editorial John Wiley \& Sons, Ltd.

Rocon E, Belda-Lois JM, Ruiz AF, Manto M, Pons JL. 2007. Design and Validation of a Rehabilitation Robotic Exoskeleton for Tremor Assessment and Suppression. IEEE Transactions on Neural Systems and Rehabilitation Engineering, 15(3):367378.

Ruiz AF, Forner-Cordero A, Rocon E, Pons JL. 2006. Exoskeletons for Rehabilitation and Motor Control. Proceedings of the IEEE International Conference on Biomedical Robotics and Biomechatronics (BioRob). 
Schiele A, Visentin G. 2003. The ESA Human Arm Exoskeleton for Space Robotics Telepresence. International Symposium on Artificial Intelligence, Robotics and Automation in Space (ISAIRAS).

Scott SH. 1999. Apparatus for measuring and perturbing shoulder and elbow joint positions and torques during reaching. $\mathrm{J}$ Neurosci. Meth. 89(2):119-127.

Scott SH. 2005. Conceptual Frameworks for Interpreting Motor Cortical Function: New Insights from a Planar MultipleJoint Paradigm in Motor Cortex In Voluntary Movements, Riehle, A., and Vaadia, E. Eds. CRC Press, London, pp. 157180.

Shadmehr R, Mussa-Ivaldi FA. 1994. Adaptative representation of dynamics during learning of a motor task. J. Neurosci. 14(1):3208-3224.

Stroeve S. 1999. Impedance characteristics of a neuromusculoskeletal model of the human arm I. Posture control. Biological Cybernetics, 81(5-6):475-494.

Tanaka Y, Onishi T, Tsuji T, Yamada N, Takeda Y, Masamori I. 2007. Analysis and Modeling of Human Impedance Properties for Designing a Human-Machine Control System. IEEE Inter- national Conference on Robotics and Automation, pp. 36273632 .

Tsagarakis NG, Caldwell D. 2003. Development and Control of a "Soft-Actuated" Exoskeleton for Use in Physiotherapy and Training. Autonomous Robots, 15:21-33.

Tsuji T, Morasso PG, Ito K. 1995. Human hand impedance characteristics during maintained posture. Biological Cybernetics, 72(6):475-485.

van der Helm FCT, Schouten AC, de Vlugt E, Brouwn GG. 2002. Identification of intrinsic and reflexive components of human arm dynamics during postural control. J Neurosci. Meth. 119(1):1-14.

de Vlugt E, Schouten AC, Van der Helm FC, Teerhuis PC, Brouwn GG. 2003. A force-controlled planar haptic device for movement control analysis of the human arm. J Neurosci. Meth. 129(1):151-168.

Zhang LQ, Rymer WZ. 1997. Simultaneous and Non-Linear Identification of Mechanical and Reflex Properties of Human Elbow Joint Muscles. IEEE Transactions On Biomedical Engineering, 44(12): 1192-1209. 

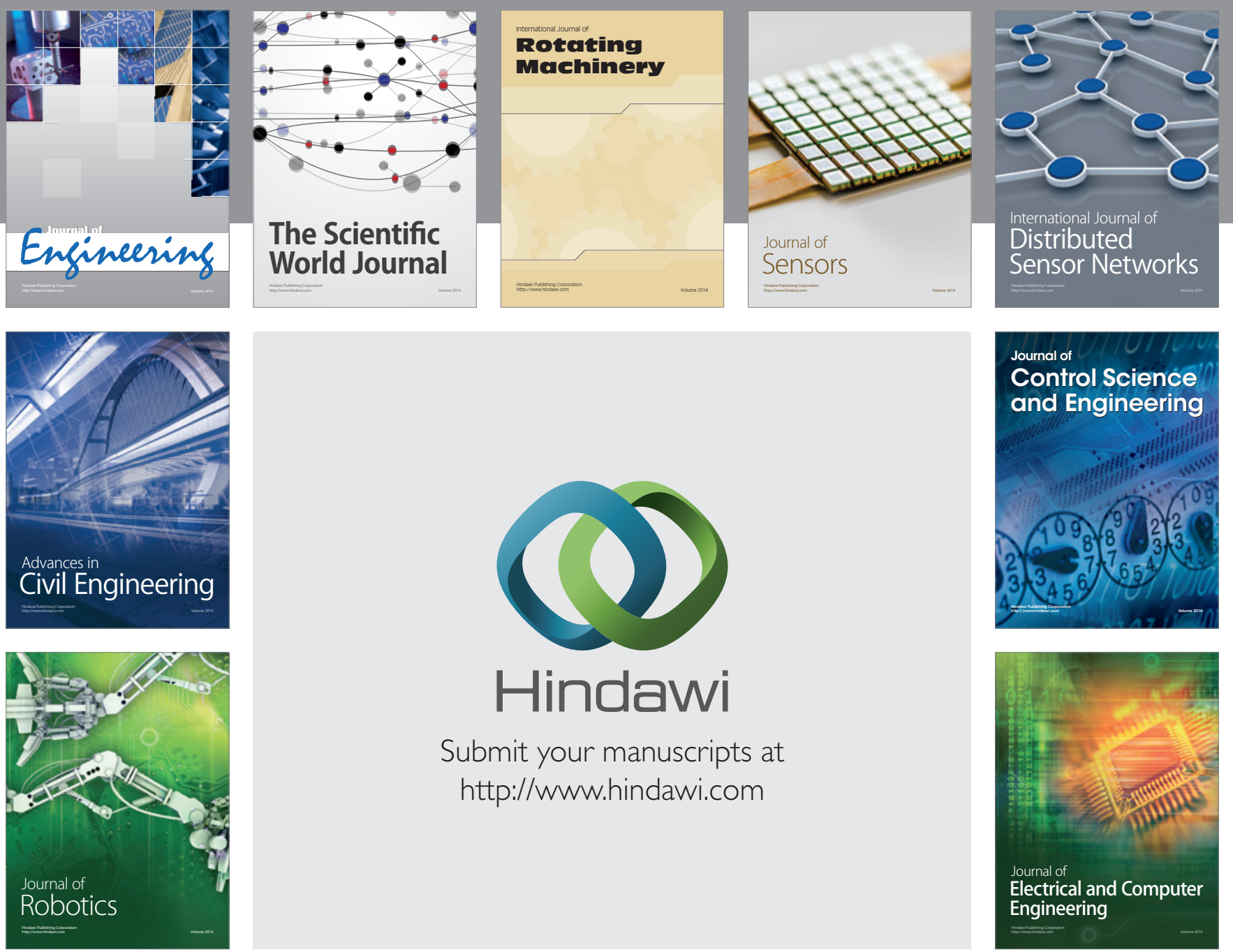

Submit your manuscripts at

http://www.hindawi.com
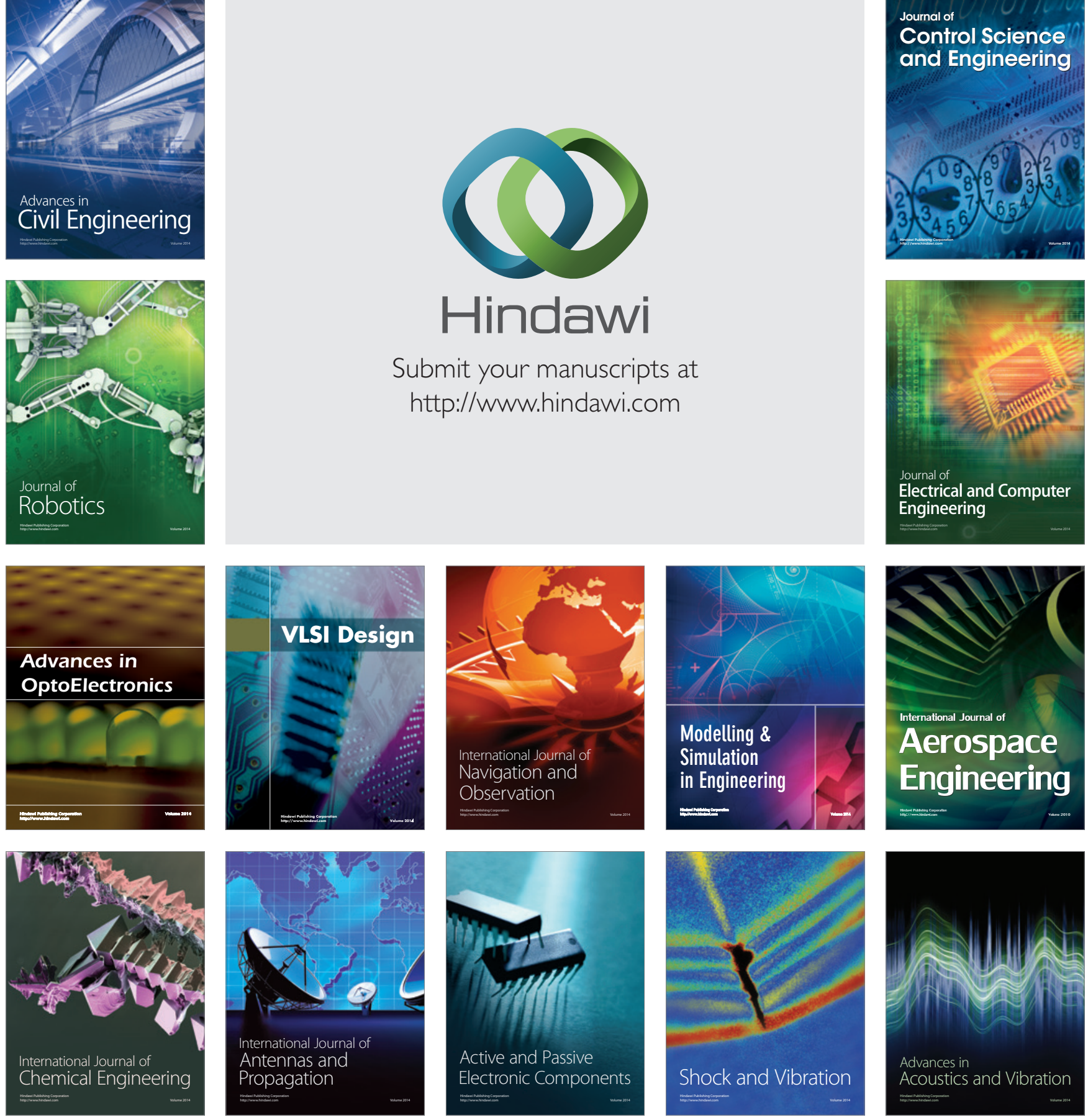